\title{
Non-invasive Screening of Diabetes Risk by Assessing Abnormalities of Sudomotor Function
}

\author{
Authors \\ G. Müller ${ }^{1}$, J. Olschewski ${ }^{1}$, T. Stange ${ }^{1}$, V. T. Hjellset ${ }^{2}$, S. Bornstein ${ }^{1}$, P. E. H. Schwarz ${ }^{1,3}$ \\ Affiliations \\ ${ }^{1}$ University Clinic Carl Gustav Carus at the Technical University Dresden, Germany \\ ${ }^{2}$ University of Oslo, Institute of General Practice and Community Medicine, Department of Preventive Medicine and \\ Epidemiology, Oslo, Norway \\ ${ }^{3}$ Paul Langerhans Institute Dresden, German Center for Diabetes Research (DZD), Dresden, Germany
}

Key words

- sudomotor function

- insulin resistance

received 03.07.2013

first decision 03.09.2013

accepted $\quad$ 10.09.2013

\section{Bibliography}

DOI http://dx.doi.org/

10.1055/s-0033-1357128

Published online:

May 5, 2014

Exp Clin Endocrinol Diabetes

2015; 123: 34-38

(C) ]. A. Barth Verlag in

Georg Thieme Verlag KG

Stuttgart · New York

ISSN 0947-7349

\section{Correspondence}

\section{Prof. Dr. P. E. H. Schwarz}

Department for Prevention and

Care of Diabetes

Medical Clinic III

University Clinic Carl Gustav

Carus at the Technical

University Dresden

Fetscherstrasse 74

01307 Dresden

Tel.: + 49/351/458 2715

Fax: $+49 / 351 / 4587319$

peter.schwarz@uniklinikum-

dresden.de

\section{Abstract \\ $\nabla$}

Background: The early detection of diabetes, and subsequent lifestyle intervention, may reduce the burden of diabetes and its complications. Several studies have identified a link between sudomotor dysfunction, insulin resistance, and pre-diabetes. The aim of this study was to evaluate the ability of a new non-invasive device EZSCAN evaluating sudomotor function to detect pre-diabetes in a German population at risk for diabetes.

Methods and findings: 200 German subjects at risk for diabetes (mean age $56 \pm 14$ years, BMI $28.4 \pm 5.4 \mathrm{~kg} / \mathrm{m}^{2}$ ) were measured for anthropometric data on inflammatory parameters, including high sensitivity $\mathrm{C}$ reactive protein (hs-CRP). The subjects also underwent an oral glucose tolerance test with measurements of plasma glucose, insulin, proinsulin, C-peptide and free fatty acids during $2 \mathrm{~h}$ following glucose challenge. Indexes for sensitivity to insulin were calculated: $\mathrm{S}_{\mathrm{I}}$ using minimal model, HOMA-IR and Matsuda index. Based on the measurement of electro-

\section{Introduction}

$\nabla$

Diabetes is a growing global concern that is affecting 285 million people today. By 2030, according to the IDF Diabetes Atlas, that number of people will have risen to 438 million [1], and double that if figures for metabolic syndrome or pre-diabetes are included. These statistics also reveal a disturbing trend: the increase in number of children and adolescents developing type 2 diabetes. Major prevention trials have shown that diabetes can be delayed or prevented in individuals with impaired glucose tolerance (IGT) using lifestyle intervention or medication [2]. However, incorporating the evidence from trials into clinical practice represents one of the major challenges for public health and clinical care. chemical sweat conductance, subjects were classified as no risk, moderate risk or high risk. According to this risk model classification, a significant difference was observed between OGTT-1 h $(p=0.004)$, AUC glucose $(p=0.011)$, AUC C-peptide $(p<0.001)$, HOMA-IR $(p=0.009)$, Matsuda $(p=0.002), S_{I}(p<0.001)$ and hs-CRP $(p=0.025)$ after adjustment for age. Among the 54 subjects with impaired fasting glucose or impaired glucose tolerance according to WHO classification, 37 had a moderate risk and 15 a high risk according to the EZSCAN risk model classification. Among the 12 subjects with newly diagnosed diabetes, 2 had a moderate risk and 10 a high risk according to the risk model classification. No adverse event was reported during or after the study.

Conclusions: These results, in accordance with a previous study performed in India, show that EZSCAN could be developed as a screening tool for diabetes risk, and could help to improve diabetes screening strategies. Results obtained from an at-risk population would have to be confirmed in a larger population.

Currently an evidence-based guideline for the prevention of type 2 diabetes [3] and a practice tool kit for the implementation of programs for the prevention of chronic metabolic diseases [4], especially diabetes mellitus, have been developed to promote improved patient compliance. There is growing evidence that interventions to prevent diabetes from developing are more efficient when applied earlier in subjects identified at risk for normoglycemic, exhibiting insulin resistance but not hyperglycemic.

Diagnosing type 2 diabetes and screening for the different pre-diabetic stages of risk require often resource-intensive measurements of a set of biomarkers, which are yet to be universally standardized. Attempts to employ questionnaire-based risk scores, for which there is some valid predictive 
efficacy, are unfortunately limited in the practical sense. An added value to clinical practice would be to have a fast, non-invasive, and reproducible diagnostic method to efficiently identify those with increased diabetes risk.

Presently, several methods - including risk models based on $\mathrm{HbA}_{1 \mathrm{c}}[5,6]$ and 1-h post-load glucose values [7] - are employed in diagnosing diabetes and pre-diabetes. However, debate continues over which single tool is ideally suited for assessing diabetes risk or identifying individuals with increased metabolic risk factors in a population-based public health setting. In general, measurements that require blood sampling are time consuming, have low sensitivity and cannot be used for screening of large population. On the other hand, questionnaire-based methods used for early screening which require individual data such as age, BMI, and blood pressure [8-10], have their own limitations. Clearly a simple-to-use and effective diagnostic or screening tool is needed for preventive intervention programs where overall cost and population receptivity are overriding factors. Diabetic neuropathy is a known complication of diabetes mellitus and manifests as impairment of nerve function due to hyperglycemia and insulin resistance [11]. To understand diabetic neuropathy development, several studies have explored sudomotor dysfunction by using quantitative sudomotor axon reflex test (QSART) or sympathetic skin response $[12,13]$. These studies have shown a direct link between impairment of sudomotor function and insulin resistance and pre-diabetes $[14,15]$. Skin biopsies confirmed damage to the sympathetic $\mathrm{C}$ nerve fibers innervating sweat glands in hyperglycemic and insulin resistance conditions; while, on the contrary, the positive effects of lifestyle intervention led to the rejuvenation of those fibers [16]. However, skin biopsies and traditional tests used to assess sudomotor function are time-consuming and/or invasive and are not efficient for screening.

EZSCAN $^{\circledR}$ (Acronym for “Easy Scan”) was recently developed to perform a noninvasive evaluation of sweat gland function through measurement of electrochemical skin conductance, based on an electrochemical reaction between sweat chloride and nickel electrodes in contact with palms of the hands and soles of the feet [17-21]. A risk model based on hand and foot conductance values adjusted for age and BMI was developed and evaluated in both controls and patients with diabetes, to predict insulin resistance and current diabetes. This predictive model was tested and evaluated in Indian and Chinese subjects at risk of diabetes according to ADA criteria [22,23], which approved the risk model in persons with normal glucose tolerance and diabetes mellitus. Persons with IGT presented a unique picture and expressed repeatedly elevated foot conductance. This was explained by the presence of an inflammatory process influencing sweat gland innervation, previously explored through skin biopsies performed in patients with pre-diabetes [24]. The risk model had to be adjusted using relative values of hand and foot conductances and systolic blood pressure.

The aim of this study was to assess the ability of this new risk model previously validated in India to detect pre-diabetes or diabetes in a German population at risk for these conditions.

\section{Methods}

$\nabla$

\section{Participants and biochemical measurements}

200 subjects from the city of Dresden and adjoining areas were recruited in the Division for Prevention and Care of Diabetes mellitus as previously described [25]. Written informed consent was obtained from all subjects and all subjects were able to give the consent themselves. Clinical investigations were conducted according to the principles expressed in the Declaration of Helsinki. Ethical approval was obtained by the University of Dresden (approval No.198062010). Most of the subjects came from German families with a family history of type 2 diabetes, obesity, or dyslipoproteinaemia. The average age of the subjects was $56 \pm 14$ years; average BMI was $28.4 \pm 5.4 \mathrm{~kg} / \mathrm{m}^{2}$. Exclusion criteria were: known diabetes mellitus, severe renal disease, disease with a strong impact on life expectancy, and therapy with drugs known to influence glucose tolerance (thiazide diuretics, beta blockers, steroids) or pregnancy of the proband. Anthropometric data (body weight, body height, BMI, waist circumference), $\mathrm{HbA}_{1 \mathrm{C}}$, lipids (triglycerides, total cholesterol, HDL-C, LDL-C) and inflammatory parameters including hs-CRP were recorded. All individuals underwent a $75 \mathrm{~g}$ oral glucose tolerance test following an overnight period of fasting ( $10 \mathrm{~h}$ minimum) with measurements of plasma glucose, insulin, proinsulin, C-peptide and free fatty acids (NEFA) at fasting and at 30,60, 90 and $120 \mathrm{~min}$ after glucose challenge. The cohort was divided into 4 glucose tolerance groups according to the results of the baseline and follow-up OGTT: normoglycemic (NGT), impaired glucose tolerance (IGT), those with impaired fasting glucose (IFG), and type 2 diabetes mellitus based on the WHO criteria of 1997, which was previously reported [25-27].

\section{Insulin sensitivity, Matsuda index, HOMA-IR}

The Minimal model was used to calculate insulin sensitivity $\left(\mathrm{S}_{\mathrm{I}}\right)$ index based on the following formula [28].

$$
S_{\mathrm{IOGTT})}=\frac{f \cdot D_{\text {OGTT }} \cdot \frac{\operatorname{AUC}[\Delta G(t) / G(t)]}{\mathrm{AUC}[\Delta G(t)]}-G E \cdot A U C[\Delta G(t) / G(t)]}{\mathrm{AUC}[\Delta I(t)]}
$$

Where $G$ is plasma glucose concentration; $\Delta G$ and $\Delta I$ are glucose and insulin concentrations above basal, respectively; AUC denotes the area under the curve calculated from time 0 to $t \rightarrow \infty$, GE is glucose effectiveness ( $\left.\mathrm{dl} . \mathrm{kg}^{-1} \mathrm{~min}^{-1}\right)$; DOGTT is the dose of ingested glucose per unit of body weight $(\mathrm{mg} / \mathrm{kg})$; and $f$ is the fraction of ingested glucose that actually appears in the systemic circulation. Calculation of $S_{\mathrm{I}}$ requires insertion of values for GE and $f$. Values proposed by Monti et al. were used: $\mathrm{GE}=0.024 \mathrm{dl} . \mathrm{kg}^{-1} \cdot \mathrm{min}^{-1}$ and $f=0.8$ [29]. Homeostasis model assessment - insulin resistance (HOMA-IR) and Matsuda index were also calculated.

\section{Measurement of sweat function}

EZSCAN, a patented device designed to perform a precise evaluation of sweat gland function through reverse iontophoresis and chrono-amperometry, has been described previously $[17,20]$. The apparatus consists of 2 sets of large area nickel electrodes for the hands and the feet, which are connected to a computer for recording and data management purposes; electrodes are used alternatively as an anode or a cathode and a direct current (DC) incremental voltage $\leq 4$ volts is applied on the anode. To conduct the test, patients are required to place their hands and feet on the electrodes and then to stand still for $2 \mathrm{~min}$. Through reverse iontophoresis, the device generates a voltage on the cathode and a current (intensity of about $0.2 \mathrm{~mA}$ ) between the anode and the cathode, proportional to electrochemical reaction between chlorides of the sweat and nickel electrodes. At low voltages, less than $10 \mathrm{~V}$, the stratum corneum is electrically insulating and only sweat gland ducts are conductive [30]. The electrochemical sweat 
conductance (ESC), expressed in microSiemens $(\mu \mathrm{S})$, is the ratio between current generated and the constant DC stimulus applied on electrodes. Patients were classified according to their risk score using a color classification allowing easy patient understanding green: no risk, yellow: moderate risk and orange/red: high risk.

\section{Statistical analyses}

Results for quantitative variables are shown as mean values \pm standard deviations (SD). Log transformation was performed for variables not normally distributed. Group means were globally compared using student t-test. Pearson Chi-square or Fisher's exact tests were used for the comparisons of percentages. Analysis of variance (ANOVA) and logistic regression, adjusted for age, have been performed to compare quantitative variables and percentages. The statistical analysis was done using R 2.13.1 (The R-project for statistical computing) [31]. As a rule, a p-value $<0.05$ was regarded as statistically significant.

\section{Results}

$\nabla$

Of the 200 men and women included in the study initially, 134 (67\%) were diagnosed with NGT; 54 (27\%) exhibited IGT/IFG; and 12 (6\%) were newly diagnosed with type 2 diabetes according to WHO classification. Among the 54 subjects with IFG/IGT, 37 had a moderate EZSCAN risk score and 15 a high risk according to risk model classification based on sudomotor function assessment, whereas lower values mean lower risk and vice versa. Among the 12 subjects with diabetes, 2 had a moderate risk and 10 a high risk according to risk model classification. Considering subjects with no risk vs. subjects with moderate or high risk, sensitivity to diagnose IFG/IGT or diabetes was $97 \%$ and specificity $31 \%$ with a negative predictive value of $95 \%$. Demography and main results according to risk classification based on sudomotor dysfunction assessments are displayed in 0 Table 1. After adjustment for age a significant difference was observed between each risk model group in AUC glucose $(\mathrm{p}=0.011)$, AUC C-peptide ( $\mathrm{p}<0.001)$, HOMA-IR $(\mathrm{p}=0.009)$, Matsuda $(p=0.002), S_{I}(p<0.001)$ and hs-CRP $(p=0.025)$. No association was detected between the risk model and the patient lipid profile. Blood concentrations during $2 \mathrm{~h}$-OGTT for glucose, insulin, pro-insulin, C-peptide and free fatty acids are displayed on $\bullet$ Fig. 1.

Mean EZSCAN risk score values for NGT, IFG/IGT and diabetes groups were $48 \pm 26,57 \pm 15$ and $66 \pm 12$, respectively.

No adverse event was reported during or after the study. More specifically, no patient perceived the voltage applied or the current produced. No reddening of the palms or soles was observed.

\section{Discussion}

\section{$\nabla$}

The EZSCAN technology was evaluated in this study as a potential screening tool to detect diabetes risk. This technology has several advantages: reproducibility, non-invasiveness, and short test duration allowing numerous follow-up tests with very low supply costs.

This study, performed in a population of German subjects at risk for pre-diabetes or diabetes, shows that non-invasive assessment of sudomotor function could present an effective method to identify people with increased diabetes risk. The assessment of sweat function based on an electrochemical reaction between sweat chloride and nickel electrodes allows a valid detection of patients with insulin resistance or type 2 diabetes, with a very low rate of false negative test results.

It is known that long diabetes duration and inadequately managed diabetes treatment increase the risk of developing neuropathy. Neuropathy diagnosed in a clinical setting is often in its late, irreparable stages, due to the diagnostic tools involved (biothesiometer or turning fork measuring large fiber damages), and has already manifested as significant impairment of nerve function [32,33]. From a clinical point of view there is only little known about very early stages of autonomic nerve function impairment as it relates to the development of insulin resistance. Recent studies have shown a link in patients with pre-diabetes between more severe stages of insulin resistance and peripheral autonomic nerve fiber impairment $[15,34]$ and also and association in childhood obesity were increased BMI and elevated HOMA-IR is associated with impaired autonomic function [35]. In addition a recent study evidenced that insulin resistance itself was independently associated with peripheral and autonomic neuropathy [36]. Impairment of these autonomic sympathetic $C$ nerve fibers innervating sweat glands leads to sudomotor dysfunction. Applying electrodes with a current voltage inducing current linked to an electrochemical reaction between sweat chloride and electrodes allows a precise evaluation of the sweat dysfunction. The measured conductance (ratio of the current and voltage) is directly related to the impairment of the $\mathrm{C}$ nerve fibers innervating autonomic sweat glands. A proof of concept study has shown good correlation between conductance and sweat chloride concentration as measured by a sweat test in controls and patients with cystic fibrosis [17]. This is in conjunction with a current observational study showing that insulin treatment improves the neuropathy measured by EZSCAN [33]. The advantage of this technology, EZSCAN, is that it is non-invasive, can be performed quickly (in less than $3 \mathrm{~min}$ ), and will allow qualitative assessment of pre-diabetic stages based on the subject's measured chloride-dependent conductance levels. Previous studies have presented evidence of the method's reproducibility $[33,37]$. A risk assessment model has been developed first in patients with diabetes, has undergone validation in an Indian test population at risk of diabetes [22], and has now been confirmed in different stages of pre-diabetes risk in a German-Caucasian population. Tests for insulin resistance showed a difference between subjects without risk, according to the risk model, and subjects with moderate or high risk ( $\bullet$ Table 1). A significant difference could be seen in hs-CRP (a marker of inflammation) between subjects with moderate risk and subjects with no risk, in accordance with the inflammation process observed in sweat gland innervation of subjects with IGT [23]. This hypothesis has to be confirmed by assessment of other markers of inflammation. These data help to further support the EZSCAN risk score as a technology that may meet requirements for the screening of metabolic risk stages. EZSCAN could be developed as an alternative to other biomarker-based medical risk assessment strategies or questionnaire-based public health screening strategies.

Several limitations warrant interpretation of the study results. First, the patients were not selected randomly, and were recruited based on their existing increased risk of diabetes. This may generate a bias - potentially an underestimation of the EZSCAN results. The second limitation of our study is that the association was only tested against the EZSCAN-developed risk model and not vice versa. In particular, investigating the differences 
Table 1 Demography and main results according to EZSCAN risk model classification.

\begin{tabular}{|c|c|c|c|c|c|c|}
\hline & Overall & $\begin{array}{l}\text { No risk } \\
\text { (Green) }\end{array}$ & $\begin{array}{l}\text { Moderate risk } \\
\text { (Yellow) }\end{array}$ & $\begin{array}{l}\text { High risk } \\
\text { (Orange/red) }\end{array}$ & $\mathbf{P}$ & P adj. ${ }^{* *}$ \\
\hline & $n=200$ & $n=43$ & $\mathrm{n}=86$ & $n=71$ & & \\
\hline age (yrs) & $56 \pm 14$ & $37 \pm 11$ & $58 \pm 10$ & $66 \pm 8$ & $<0.001$ & - \\
\hline gender (n men, \%) & $92(46 \%)$ & $22(51 \%)$ & $36(42 \%)$ & $34(48 \%)$ & NS & - \\
\hline BMI $\left(\mathrm{kg} / \mathrm{m}^{2}\right)$ & $28.4 \pm 5.4$ & $25.4 \pm 3.9$ & $29.4 \pm 5.9$ & $29.1 \pm 5.1$ & $<0.001$ & $<0.001$ \\
\hline waist (cm) & $97.0 \pm 13.3$ & $88.8 \pm 10.8$ & $99.2 \pm 14.1$ & $99.3 \pm 11.9$ & $<0.001$ & $<0.001$ \\
\hline $\mathrm{SBP}(\mathrm{mm} \mathrm{Hg})$ & $135 \pm 16$ & $124 \pm 13$ & $138 \pm 16$ & $139 \pm 16$ & $<0.001$ & 0.019 \\
\hline $\mathrm{HbA}_{1 \mathrm{c}}(\%)$ & $5.7 \pm 0.5$ & $5.3 \pm 0.3$ & $5.7 \pm 0.4$ & $5.8 \pm 0.5$ & $<0.001$ & 0.098 \\
\hline $\mathrm{FPG}(\mathrm{mmol} / \mathrm{L})$ & $5.3 \pm 0.7$ & $4.9 \pm 0.5$ & $5.4 \pm 0.6$ & $5.5 \pm 0.9$ & $<0.001$ & 0.085 \\
\hline OGTT-1h (mmol/L) & $9.3 \pm 2.6$ & $7.7 \pm 2.1$ & $9.6 \pm 2.4$ & $10.0 \pm 2.6$ & $<0.001$ & 0.004 \\
\hline OGTT-2h (mmol/L) & $6.9 \pm 2.3$ & $5.5 \pm 1.6$ & $7.2 \pm 2.1$ & $7.4 \pm 2.5$ & $<0.001$ & 0.080 \\
\hline fasting free fatty acid (mmol/L) & $0.52 \pm 0.23$ & $0.44 \pm 0.20$ & $0.52 \pm 0.21$ & $0.57 \pm 0.25$ & 0.009 & NS \\
\hline total cholesterol (mmol/L) & $5.4 \pm 1.0$ & $5.1 \pm 1.1$ & $5.4 \pm 0.9$ & $5.6 \pm 0.9$ & 0.008 & NS \\
\hline triglycerides (mmol/L) & $1.4 \pm 0.9$ & $1.3 \pm 0.9$ & $1.4 \pm 1.0$ & $1.4 \pm 0.8$ & NS & - \\
\hline HDLC (mmol/L) & $1.5 \pm 0.4$ & $1.5 \pm 0.4$ & $1.5 \pm 0.4$ & $1.6 \pm 0.4$ & NS & - \\
\hline $\mathrm{LDLC}(\mathrm{mmol} / \mathrm{L})$ & $3.3 \pm 0.9$ & $3.1 \pm 1.0$ & $3.3 \pm 0.8$ & $3.5 \pm 0.8$ & 0.054 & NS \\
\hline hs-CRP (mg/L) & $2.2 \pm 3.1$ & $1.6 \pm 2.3$ & $2.0 \pm 2.2$ & $2.7 \pm 4.1$ & 0.015 & 0.025 \\
\hline AUC glucose 120 (mmol/Lmn) & $983 \pm 221$ & $836 \pm 174$ & $1009 \pm 202$ & $1041 \pm 233$ & $<0.001$ & 0.011 \\
\hline AUC insulin-120 (mmol/Lmn) & $60698 \pm 39334$ & $52141 \pm 33466$ & $65595 \pm 42412$ & $60008 \pm 38342$ & 0.061 & 0.010 \\
\hline AUC pro-insulin-120 (mmol/Lmn) & $3245 \pm 2560$ & $3185 \pm 2679$ & $3394 \pm 2810$ & $3102 \pm 2164$ & NS & - \\
\hline AUC C-peptide-120 (mmol/Lmn) & $103 \pm 37$ & $78 \pm 31$ & $111 \pm 40$ & $107 \pm 31$ & $<0.001$ & $<0.001$ \\
\hline HOMA-IR & $2.9 \pm 2.4$ & $2.0 \pm 1.5$ & $3.2 \pm 2.6$ & $3.0 \pm 2.5$ & 0.028 & 0.009 \\
\hline MATSUDA & $4.4 \pm 2.9$ & $5.9 \pm 3.8$ & $3.9 \pm 2.5$ & $4.0 \pm 2.4$ & 0.001 & 0.002 \\
\hline$S_{1}$ & $8.8 \pm 8.2$ & $14.5 \pm 11.4$ & $7.3 \pm 6.4$ & $7.3 \pm 6.3$ & $<0.001$ & $<0.001$ \\
\hline mean hands conductances $(\mu S)$ & $66 \pm 16$ & $74 \pm 9$ & $69 \pm 14$ & $58 \pm 18$ & $<0.001$ & $<0.001$ \\
\hline mean feet conductances $(\mu S)$ & $78 \pm 11$ & $85 \pm 6$ & $83 \pm 6$ & $70 \pm 13$ & $<0.001$ & $<0.001$ \\
\hline ratio (feet/hands) & $1.26 \pm 0.33$ & $1.17 \pm 0.16$ & $1.26 \pm 0.32$ & $1.30 \pm 0.40$ & 0.100 & NS \\
\hline $\operatorname{NGT}(\mathrm{n}, \%)$ & $134(67 \%)$ & $41(95 \%)$ & $47(55 \%)$ & $46(65 \%)$ & $<0.001$ & $<0.001$ \\
\hline IFG/IGT (n, \%) & 54 (27\%) & $2(5 \%)$ & $37(43 \%)$ & $15(21 \%)$ & $<0.001$ & $<0.001$ \\
\hline diabetes (n, \%) & $12(6 \%)$ & $0(0 \%)$ & $2(2 \%)$ & $10(14 \%)$ & 0.002 & $<0.001$ \\
\hline
\end{tabular}

**Adjusted on age
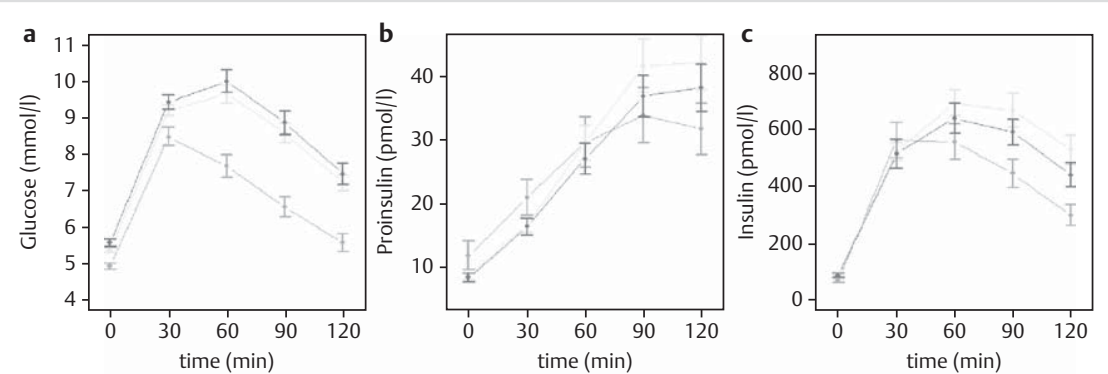

Fig. 1 AUC120 for Glucose a, insulin b, proinsulin c, C-Peptide $\mathbf{d}$ and free fatty acid e according to risk classification defined by EZSCAN. Green: no risk, yellow: moderate risk and red: high risk.

Values are mean \pm SEM.
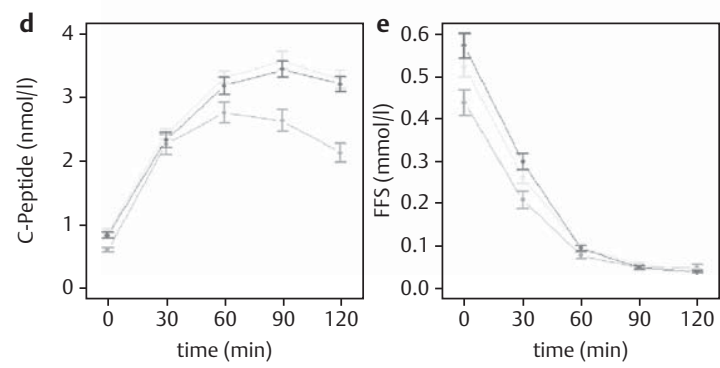

between patients with IFG or IGT would be an interesting topic to further evaluate the risk model against clinical patients' profiles. Therefore, it is necessary to validate the association between the risk model and insulin resistance in a randomized study, and also in larger populations. In addition a follow-up study seems necessary to assess the predictive power of the method.
The EZSCAN provides an interesting new technology for screening. The impairment of autonomic sudomotor dysfunction can be used as indicator to identify people with high risk for diabetes and maybe other metabolic and cardiovascular diseases. Recently, this technology is applicable to adult population, but the concept of impaired autonomic dysfunction is also visible in obese insulin resistant children. The validity of this technology needs to be 
further investigated: it is part of the large European prospective trial ePREDICE that just begins. Summarizing this, the EZSCAN provides an interesting non-invasive technology for screening of chronic diseases in various settings.

Conflict of interest statement: This work was funded by Impeto Medical, 17 rue Campagne Première, 75014 Paris, France.

\section{References}

1 Idf. Diabetes Atlas. Fourth Edition. Brussels: International Diabetes Federation, 2009

2 Schwarz PE, Greaves CJ, Lindstrom J et al. Nonpharmacological interventions for the prevention of type 2 diabetes mellitus. Nature reviews Endocrinology 2012; 8: 363-373 doi:10.1038/nrendo.2011.232

3 Paulweber B, Valensi P, Lindström J et al. A European evidence-based guideline for the prevention of type 2 diabetes. Horm Metab Res 2010; 42: S3-S36

4 Lindstrom J, Neumann A, Sheppard KE et al. Take action to prevent diabetes - the IMAGE toolkit for the prevention of type 2 diabetes in Europe. Hormone and metabolic research =Hormon- und Stoffwechselforschung = Hormones et metabolisme 2010; 42 (Suppl 1): S37-S55 doi:10.1055/s-0029-1240975

5 Ziemer DC, Kolm P, Weintraub WS et al. Glucose-independent, blackwhite differences in hemoglobin A1c levels: a cross-sectional analysis of 2 studies. Ann Intern Med 152: 770-777

6 Bergman $M$. Inadequacies of absolute threshold levels for diagnosing prediabetes. Diabetes Metab Res Rev 26: 3-6

7 Abdul-Ghani MA, Lyssenko V, Tuomi T et al. Fasting versus postload plasma glucose concentration and the risk for future type 2 diabetes: results from the Botnia Study. Diabetes care 2009; 32: 281-286

8 Lindstrom J, Tuomilehto J. The Diabetes Risk Score: A practical tool to predict type 2 diabetes risk. Diabetes care 2003; 26: 725-731

9 Schwarz PE, Li J, Lindstrom J et al. Tools for predicting the risk of type 2 diabetes in daily practice. Hormone and metabolic research $=$ Hormon- und Stoffwechselforschung=Hormones et metabolisme 2009; 41: 86-97

10 Schwarz PE, Li J, Reimann $M$ et al. The Finnish Diabetes Risk Score Is Associated with Insulin Resistance and Progression towards Type 2 Diabetes. The Journal of clinical endocrinology and metabolism 2009; 94: 920-926

11 Kim B, McLean LL, Philip SS et al. Hyperinsulinemia induces insulin resistance in dorsal root ganglion neurons. Endocrinology 2011; 152: 3638-3647

12 Low PA. Evaluation of sudomotor function. Clin Neurophysiol 2004; 115: $1506-1513$

13 Provitera $V$, Nolano $M$, Caporaso $G$ et al. Evaluation of sudomotor function in diabetes using the dynamic sweat test. Neurology 2010; 74: 50-56

14 Gibbons $\mathrm{CH}$, Illigens BM, Wang $N$ et al. Quantification of sudomotor innervation: a comparison of three methods. Muscle \& nerve 2010; 42: $112-119$

15 Grandinetti A, Chow DC, Sletten DM et al. Impaired glucose tolerance is associated with postganglionic sudomotor impairment. Clinical autonomic research: official journal of the Clinical Autonomic Research Society 2007; 17: 231-233

16 Smith AG, Russell J, Feldman EL et al. Lifestyle intervention for prediabetic neuropathy. Diabetes care 2006; 29: 1294-1299

17 Hubert D, Brunswick $P$, Calvet JH et al. Abnormal electrochemical skin conductance in cystic fibrosis. Journal of cystic fibrosis: official journal of the European Cystic Fibrosis Society 2011; 10: 15-20 doi:10.1016/j. jcf.2010.09.002

18 Ayoub $H$, Griveau S, Lair $V$ et al. Electrochemical characterization of nickel electrodes in phosphate and carbonate electrolytes in view of assessing a medical diagnostic device for the detection of early diabetes. Electroanalysis 2010; 22: 2483-2490
19 Brunswick P, Mayaudon H, Albin Vet al. Use of Ni electrodes chronoamperometry for improved diagnostics of diabetes and cardiac diseases. Conference proceedings: Annual International Conference of the IEEE Engineering in Medicine and Biology Society Conference 2007; 2007: 4544-4547 doi:10.1109/iembs.2007.4353350

20 Mayaudon H, Miloche PO, Bauduceau B. A new simple method for assessing sudomotor function: relevance in type 2 diabetes. Diabetes \& metabolism 2010; 36: 450-454

21 Ayoub H, Griveau S, Lair V et al. Electrochemical characterization of nickel electrodes in phosphate and carbonate electrolytes in view of assessing a medical diagnostic device for the detection of early diabetes. Electroanalysis 2012; 24: 386-391

22 Ramachandran A, Moses A, Shetty Set al. A new non-invasive technology to screen for dysglycaemia including diabetes. Diabetes research and clinical practice 2010; 88: 302-306 doi:10.1016/j.diabres.2010.01.023

23 Sheng CS, Zeng WF, Huang QF et al. Accuracy of a Novel Non-Invasive technology based EZSCAN system for the diagnosis of diabetes mellitus in Chinese. Diabetol Metab Syndr 2011; 3: 36 doi:10.1186/17585996-3-36

24 Lacomis D. Small-fiber neuropathy. Muscle \& nerve 2002; 26: 173-188

25 Schwarz PE, Towers GW, Fischer S et al. Hypoadiponectinemia is associated with progression toward type 2 diabetes and genetic variation in the ADIPOQ gene promoter. Diabetes care 2006; 29: 1645-1650 doi:10.2337/dc05-2123

26 Paulweber B, Valensi P, Lindstrom J et al. A European evidence-based guideline for the prevention of type 2 diabetes. Hormone and metabolic research $=$ Hormon- und Stoffwechselforschung $=$ Hormones et metabolisme 2010; 42(Suppl 1):S3-S36 doi:10.1055/s-0029-1240928

27 Abdul-Ghani MA, Abdul-Ghani T, Muller G et al. Role of glycated hemoglobin in the prediction of future risk of T2DM. The Journal of clinical endocrinology and metabolism 2011; 96: 2596-2600 doi:10.1210/ jc.2010-1698

28 Breda E, Cavaghan MK, Toffolo $G$ et al. Oral glucose tolerance test minimal model indexes of beta-cell function and insulin sensitivity. Diabetes 2001; 50: 150-158

29 Monti LD, Poma R, Caumo A et al. Intravenous infusion of diarginylinsulin, an insulin analogue: effects on glucose turnover and lipid levels in insulin-treated type II diabetic patients. Metabolism: clinical and experimental 1992; 41: 540-544

30 Chizmadzhev YA, Indenbom AV, Kuzmin PI et al. Electrical properties of skin at moderate voltages: contribution of appendageal macropores. Biophys J 1998; 74: 843-856

31 R. Development. Core. Team. R: A Language and Environment for Statistical Computing. In: http://www.R-project.org (ed.).Vienna, Austria: Foundation for Statistical Computing, 2011

32 Tesfaye S, Boulton AJ, Dyck PJ et al. Diabetic neuropathies: update on definitions, diagnostic criteria, estimation of severity, and treatments. Diabetes care 2010; 33: 2285-2293 doi:10.2337/dc10-1303

33 Calvet JH, Dupin J, Winiecki $H$ et al. Assessment of Small Fiber Neuropathy through a Quick, Simple and Non Invasive Method in a German Diabetes Outpatient Clinic. Experimental and clinical endocrinology \& diabetes: official journal, German Society of Endocrinology [and] German Diabetes Association 2013; 121: 80-83 doi:10.1055/s-0032-1323777

34 Sumner CJ, Sheth S, Griffin JW et al. The spectrum of neuropathy in diabetes and impaired glucose tolerance. Neurology 2003; 60: 108-111

35 Baum P, Petroff D, Classen J et al. Dysfunction of autonomic nervous system in childhood obesity: a cross-sectional study. PloS one 2013; 8: e54546 doi:10.1371/journal.pone.0054546

36 Lee KO, Nam JS, Ahn CW et al. Insulin resistance is independently associated with peripheral and autonomic neuropathy in Korean type 2 diabetic patients. Acta diabetologica 2012; 49: 97-103 doi:10.1007| s00592-010-0176-6

37 Schwarz PE, Brunswick P, Calvet JH. EZSCAN a new tool to detect diabetes risk. Br J Diabetes Vasc Dis 2011; 11: 204-209 